\title{
A new generalization of Weibull-exponential distribution with application
}

\author{
Ramadan A. ZeinEldin ${ }^{\mathrm{a}, \mathrm{b}}$, M. Elgarhy,* \\ ${ }^{a}$ Deanship of Scientific Research, King AbdulAziz University, Kingdom of Saudi Arabia. \\ ${ }^{b}$ Institute of Statistical Studies and Research, Cairo University, Egypt. \\ ${ }^{c}$ Vice Presidency for Graduate Studies and Scientific Research, University of Jeddah, Jeddah, Kingdom of Saudi Arabia.
}

Communicated by A. Atangana

\begin{abstract}
In this article, we will introduce a new five-parameter continuous model, called the Kumaraswamy Weibull exponential distribution based on Kumaraswamy Weibull-G family [A. S. Hassan, M. Elgarhy, Adv. Appl. Stat., 48 (2016), 205-239]. The new model contains some new distributions as well as some former distributions. Various mathematical properties of this distribution are studied. General explicit expressions for the quantile function, expansion of distribution and density functions, moments, generating function, incomplete moments, conditional moments, residual life function, reversed residual life function, mean deviation, inequality measures, Rényi and q-entropies, probability weighted moments, and order statistics are obtained. The estimation of the model parameters is discussed using maximum likelihood method. The practical importance of the new distribution is demonstrated through real data sets where we compare it with several lifetime distributions.
\end{abstract}

Keywords: Exponential distribution, Kumaraswamy Weibull-G family of distributions, moments, order statistics, maximum likelihood estimation.

2010 MSC: 60E05, 62E10, 62N05.

(C)2018 All rights reserved.

\section{Introduction}

In the last few years, new generated families of continuous distributions have attracted several statisticians to develop new models. These families have been obtained by introducing one or more additional shape parameter(s) to the baseline distribution. Some of the generated families are: the beta-G [12, 19], gamma-G (type 1) [27], Kumaraswamy-G (Kw-G) [6], McDonald-G (Mc-G) [2], gamma-G (type 2) [25], transformed-transformer (T-X) [4], Weibull-G [5], Kumaraswamy odd log-logistic [3], Garhy-G [9], exponentiated Weibull-G [14, 15] introduced a new family called Kumaraswamy Weibull-generated, The

\footnotetext{
*Corresponding author

Email addresses: rzainaldeen@kau.edu.sa (Ramadan A. ZeinEldin), m_elgarhy85@yahoo.com (M. Elgarhy)
}

doi: $10.22436 /$ jnsa.011.09.09

Received: 2018-03-04 Revised: 2018-03-27 Accepted: 2018-04-18 
additive Weibull-G [17], type II half logistic-G [10, 16] introduced exponentiated extended-G family. The cumulative distribution function (cdf) of Kumaraswamy Weibull-generated family is given by

$$
F(x)=1-\left[1-\left(1-e^{-\alpha\left[\frac{G(x)}{1-G(x)}\right]^{\beta}}\right)^{a}\right]^{b} ; x>0 ; a, b, \alpha, \beta>0,
$$

where $a, b, \beta>0$ are the three shape parameters and $\alpha>0$ is the scale parameter. The cdf (1.1) provides a wider family of continuous distributions. The probability density function (pdf) corresponding to (1.1) is given by

$$
\begin{aligned}
f(x)= & a b \alpha \beta \frac{(G(x))^{\beta-1} g(x)}{(1-G(x))^{\beta+1}} e^{-\alpha\left[\frac{G(x)}{1-G(x)}\right]^{\beta}}\left[1-e^{-\alpha\left[\frac{G(x)}{1-G(x)}\right]^{\beta}}\right]^{a-1} \\
& \times\left[1-\left(1-e^{-\alpha\left[\frac{G(x)}{1-G(x)}\right]^{\beta}}\right)^{a}\right]^{b-1}, x>0, a, b, \alpha, \beta>0 .
\end{aligned}
$$

In this paper we introduce a new five-parameter model as a competitive extension for the exponential distribution using the KwW-G family. The new distribution extends some recent distributions and provides some new distributions. The rest of the paper is outlined as follows. In Section 2, we define the Kumaraswamy Weibull exponential (KwWE) distribution and provide some special models. In Section 3, we derive a very useful representation for the $(\mathrm{K} w \mathrm{WE})$ density and distribution functions. In the same section, some general mathematical properties of the proposed distribution are derived. The maximum likelihood method is applied to drive the estimates of the model parameters in Section 4. Simulation study is carried out to estimate the model parameters of (KwWE) distribution in Section 5. Section 6 gives an illustrative example to explain how a real data set can be modeled by $\mathrm{K} w \mathrm{WE}$ and finally we conclude the paper in Section 7.

\section{The Kumaraswamy weibull-exponential}

In this section, the five-parameter $\mathrm{K} w \mathrm{WE}$ distribution is obtained based on the $\mathrm{K} w \mathrm{~W}-\mathrm{G}$ family which was explored in [14].

Let, the random variable $X$ follows the exponential distribution with pdf given by

$$
g(x ; \lambda)=\lambda e^{-\lambda x} ; \quad x>0, \lambda>0,
$$

where, $\lambda>0$ is the scale parameter.

The cdf of exponential distribution is given by

$$
\mathrm{G}(x ; \lambda)=1-e^{-\lambda x} .
$$

Substituting from pdf (2.1) and cdf (2.2) into cdf (1.1), then the cdf of Kumaraswamy Weibull exponential distribution, denoted by $\operatorname{KwWE}(a, b, \alpha, \beta, \lambda)$, takes the following form

$$
F(x ; \Psi)=1-\left[1-\left(1-e^{-\alpha\left(e^{\lambda x}-1\right)^{\beta}}\right)^{a}\right]^{b}, a, b, \alpha, \beta, \lambda>0, x>0,
$$

where $\Psi \equiv(a, b, \alpha, \beta, \lambda)$ is the set of parameters. Inserting the pdf (2.1) and cdf (2.2) into (1.2), then the pdf of $\mathrm{K} w \mathrm{WE}$ takes the following form

$$
f(x ; \Psi)=a b \alpha \beta \lambda\left[e^{\lambda x}-1\right]^{\beta-1} e^{-\left\{\alpha\left(e^{\lambda x}-1\right)^{\beta}-\lambda x\right\}}\left(1-e^{-\alpha\left(e^{\lambda x}-1\right)^{\beta}}\right)^{a-1}\left[1-\left(1-e^{-\alpha\left(e^{\lambda x}-1\right)^{\beta}}\right)^{a}\right]^{b-1} .
$$

The pdf (2.3) contains some new distributions as well as some current distributions. Table (1) lists the special sub-models of the $\mathrm{K} w \mathrm{WE}$ distribution. 
Table 1: Special models of the Kumaraswamy Weibull-exponential distribution.

\begin{tabular}{|c|c|c|c|c|c|c|c|c|}
\hline & Model & $a$ & $\mathrm{~b}$ & $\alpha$ & $\beta$ & $\lambda$ & cdf & Author \\
\hline 1 & KwEE & - & - & - & 1 & - & $\mathrm{F}(\mathrm{x})=1-\left[1-\left(1-\mathrm{e}^{-\alpha\left(\mathrm{e}^{\lambda x}-1\right)}\right)^{\mathrm{a}}\right]^{\mathrm{b}}$ & \\
\hline 2 & KwRE & - & - & - & 2 & - & 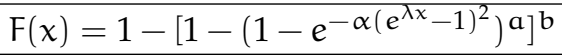 & \\
\hline 3 & EWE & - & 1 & - & - & - & $F(x)=\left(1-e^{-\alpha\left(e^{\lambda x}-1\right)^{\beta}}\right)^{a}$ & [11] \\
\hline 4 & $\mathrm{EEE}$ & - & 1 & - & 1 & - & $F(x)=\left(1-e^{-\alpha\left(e^{\lambda x}-1\right)}\right)^{a}$ & \\
\hline 5 & ERE & - & 1 & - & 2 & - & $\mathrm{F}(\mathrm{x})=\left(1-e^{\left.-\alpha\left(e^{\lambda x}-1\right)^{2}\right)^{a}}\right.$ & \\
\hline 6 & WE & 1 & 1 & - & - & - & $F(x)=1-e^{-\alpha\left(e^{\lambda x}-1\right)^{\beta}}$ & [23] \\
\hline 7 & $\mathrm{EE}$ & 1 & 1 & - & 1 & - & $\mathrm{F}(x)=1-e^{-\alpha\left(e^{\lambda x}-1\right)}$ & \\
\hline 8 & RE & 1 & 1 & - & 2 & - & $F(x)=1-e^{-\alpha\left(e^{\lambda x}-1\right)^{2}}$ & \\
\hline
\end{tabular}

The survival, hazard rate, and reversed-hazard rate functions of KwWE distribution are respectively given by

$$
\begin{aligned}
& R(x ; \Psi)=\left[1-\left(1-e^{-\alpha\left(e^{\lambda x}-1\right)^{\beta}}\right)^{a}\right]^{b}, \\
& h(x ; \Psi)=\frac{a b \alpha \beta \lambda\left[e^{\lambda x}-1\right]^{\beta-1} e^{-\left\{\alpha\left(e^{\lambda x}-1\right)^{\beta}-\lambda x\right\}}\left(1-e^{-\alpha\left(e^{\lambda x}-1\right)^{\beta}}\right)^{a-1}}{1-\left(1-e^{-\alpha\left(e^{\lambda x}-1\right)^{\beta}}\right)^{a}},
\end{aligned}
$$

and

$$
\tau(x ; \Psi)=\frac{a b \alpha \beta \lambda\left[e^{\lambda x}-1\right]^{\beta-1} e^{-\left\{\alpha\left(e^{\lambda x}-1\right)^{\beta}-\lambda x\right\}}\left(1-e^{-\alpha\left(e^{\lambda x}-1\right)^{\beta}}\right)^{a-1}\left[1-\left(1-e^{-\alpha\left(e^{\lambda x}-1\right)^{\beta}}\right)^{a}\right]^{b-1}}{1-\left[1-\left(1-e^{-\alpha\left(e^{\lambda x}-1\right)^{\beta}}\right)^{a}\right]^{b}} .
$$

Plots the pdf and hazard rate function of $\mathrm{K} w \mathrm{WE}$ distribution for some parameter values are displayed in Figures 1 and 2, respectively.

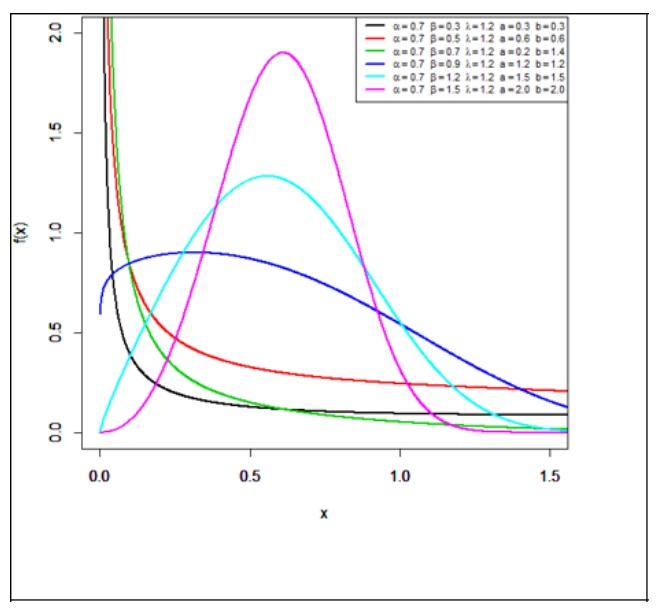

Figure 1: Plots of the pdf of the KwWE distribution for some parameter values.

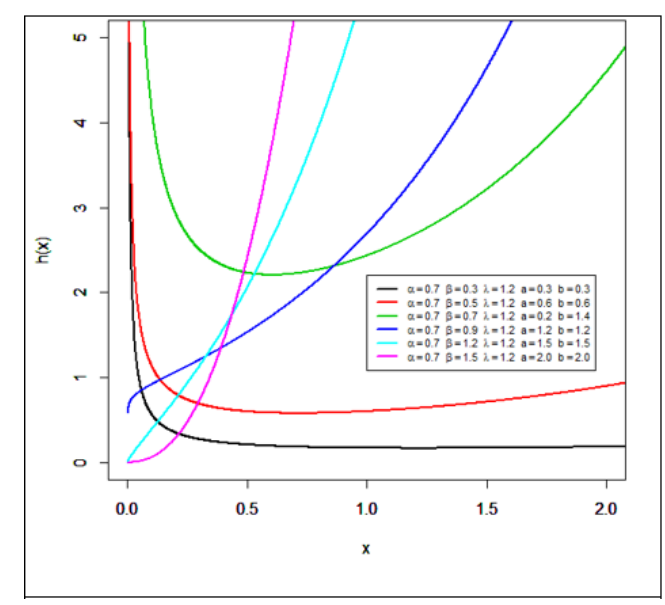

Figure 2: Plots of the hazard rate function of the KwWE distribution for some parameter values.

\section{Statistical properties}

In this section some properties of the KwWE distribution are obtained.

\subsection{Useful expansions}

In this subsection representations of the pdf and cdf for Kumaraswamy Weibull exponential distribution are derived. 
Using the generalized binomial theorem, for $\beta>0$ and $|z|<1$,

$$
(1-z)^{\beta-1}=\sum_{i=0}^{\infty}(-1)^{i}\left(\begin{array}{c}
\beta-1 \\
i
\end{array}\right) z^{i}
$$

Then, by applying the binomial theorem (3.1) in (2.3), the distribution function of $\mathrm{K} w \mathrm{~W}-\mathrm{E}$ distribution where $b>0$ becomes

$$
f(x)=\operatorname{ab} \alpha \beta \lambda\left[\frac{1-e^{-\lambda x}}{e^{-\lambda x}}\right]^{\beta-1} e^{-\left\{\alpha\left(\frac{1-e^{-\lambda x}}{e^{-\lambda x}}\right)^{\beta}-\lambda x\right\}} \sum_{i=0}^{\infty}(-1)^{i}\left(\begin{array}{c}
b-1 \\
i
\end{array}\right)\left[1-e^{-\alpha\left(\frac{1-e^{-\lambda x}}{e^{-\lambda x}}\right)^{\beta}}\right]^{a(i+1)-1} .
$$

Then, using binomial expansion again in the last equation, leads to:

$$
f(x)=\operatorname{ab} \alpha \beta \lambda\left[\frac{1-e^{-\lambda x}}{e^{-\lambda x}}\right]^{\beta-1} e^{\lambda x} \sum_{i, j=0}^{\infty}(-1)^{i+j}\left(\begin{array}{c}
b-1 \\
i
\end{array}\right)\left(\begin{array}{c}
a(i+1)-1 \\
j
\end{array}\right) e^{-\alpha(j+1)\left[\frac{1-e^{-\lambda x}}{e^{-\lambda x}}\right]^{\beta}} .
$$

Using the power series for the exponential function, we obtain

$$
e^{-\alpha(j+1)\left[\frac{1-e^{-\lambda x}}{e^{-\lambda x}}\right]^{\beta}}=\sum_{k=0}^{\infty} \frac{(-1)^{k} \alpha^{k}(j+1)^{k}}{k !}\left[\frac{1-e^{-\lambda x}}{e^{-\lambda x}}\right]^{k \beta} .
$$

Inserting this expansion (3.3) in (3.2) we have

$$
f(x)=a b \alpha \beta \lambda \sum_{i, j, k=0}^{\infty} \frac{(-1)^{i+j+k} \alpha^{k}(j+1)^{k}}{k !}\left(\begin{array}{c}
b-1 \\
i
\end{array}\right)\left(\begin{array}{c}
a(i+1)-1 \\
j
\end{array}\right) \times e^{\lambda x}\left[\frac{1-e^{-\lambda x}}{e^{-\lambda x}}\right]^{\beta(k+1)-1},
$$

we can write the last equation as

$$
f(x)=a b \alpha \beta \lambda \sum_{i, j, k=0}^{\infty} \frac{(-1)^{i+j+k} \alpha^{k}(j+1)^{k} e^{-\lambda x}}{k !}\left(\begin{array}{c}
b-1 \\
i
\end{array}\right)\left(\begin{array}{c}
a(i+1)-1 \\
j
\end{array}\right)\left[\frac{\left(1-e^{-\lambda x}\right)^{\beta(k+1)-1}}{\left(1-\left(1-e^{-\lambda x}\right)\right)^{\beta(k+1)+1}}\right],
$$

then,

$$
\begin{aligned}
f(x)= & a b \alpha \beta \lambda \sum_{i, j, k, m}^{\infty} \frac{(-1)^{i+j+k} \alpha^{k}(j+1)^{k} e^{-\lambda x}}{k !}\left(\begin{array}{c}
b-1 \\
i
\end{array}\right)\left(\begin{array}{c}
a(i+1)-1 \\
j
\end{array}\right) \\
& \times\left(\begin{array}{c}
\beta(k+1)+m \\
m
\end{array}\right)\left[1-e^{-\lambda x}\right]^{m+\beta(k+1)-1} .
\end{aligned}
$$

Now, using the binomial theorem, we can write the previous equation then, density function can be expressed as an infinite linear combination of exponential distribution, i.e.,

$$
f(x)=\sum_{i, j, k, m, \ell_{1}=0}^{\infty} \eta_{i, j, k, m, \ell_{1}} e^{-\lambda\left(\ell_{1}+1\right) x}
$$

where

$$
\begin{aligned}
\eta_{i, j, k, m, \ell_{1}}= & \frac{a b \beta \lambda \alpha^{k+1}(-1)^{i+j+k+\ell_{1}}(j+1)^{k}}{k !}\left(\begin{array}{c}
b-1 \\
i
\end{array}\right)\left(\begin{array}{c}
a(i+1)-1 \\
j
\end{array}\right) \\
& \times\left(\begin{array}{c}
\beta(k+1)+m \\
m
\end{array}\right)\left(\begin{array}{c}
m+\beta(k+1)-1 \\
\ell_{1}
\end{array}\right) .
\end{aligned}
$$

Now, for an expansion for the cumulative function we will have the following. 
Using binomial expansion for $[\mathrm{F}(\mathrm{x})]^{\mathrm{h}}$, where $\mathrm{h}$ is an integer, leads to:

Since,

$$
[F(x)]^{h}=\left[1-\left[1-\left(1-e^{-\alpha\left(\frac{1-e^{-\lambda x}}{e^{-\lambda x}}\right)^{\beta}}\right)^{a}\right]^{b}\right]^{h} .
$$

Then,

$$
[F(x)]^{h}=\sum_{g=0}^{h}(-1)^{g}\left(\begin{array}{c}
h \\
g
\end{array}\right)\left[1-\left(1-e^{-\alpha\left(\frac{1-e^{-\lambda x}}{e^{-\lambda x}}\right)^{\beta}}\right)^{\mathrm{a}}\right]^{b g} .
$$

Using binomial expansion another time, leads to

$$
[F(x)]^{h}=\sum_{g=0}^{h} \sum_{p=0}^{\infty}(-1)^{g+p}\left(\begin{array}{c}
h \\
g
\end{array}\right)\left(\begin{array}{c}
b g \\
p
\end{array}\right)\left(1-e^{-\alpha\left(\frac{1-e^{-\lambda x}}{e^{-\lambda x}}\right)^{\beta}}\right)^{a p} .
$$

Using binomial expansion again, leads to

$$
[F(x)]^{h}=\sum_{g=0}^{h} \sum_{p, q=0}^{\infty}(-1)^{g+p+q}\left(\begin{array}{c}
h \\
g
\end{array}\right)\left(\begin{array}{c}
b g \\
p
\end{array}\right)\left(\begin{array}{c}
a p \\
q
\end{array}\right) e^{-\alpha q\left(\frac{1-e^{-\lambda x}}{e^{-\lambda x}}\right)^{\beta}}
$$

Using the power series for the exponential function in the previous equation, we obtain

$$
[F(x)]^{h}=\sum_{g=0}^{h} \sum_{p, q, t=0}^{\infty} \frac{(-1)^{g+p+q+t}(\alpha q)^{t}}{t !}\left(\begin{array}{c}
h \\
g
\end{array}\right)\left(\begin{array}{c}
b g \\
p
\end{array}\right)\left(\begin{array}{c}
a p \\
q
\end{array}\right)\left[\frac{1-e^{-\lambda x}}{e^{-\lambda x}}\right]^{\beta t},
$$

we can write the last equation as

$$
[F(x)]^{h}=\sum_{g=0}^{h} \sum_{p, q, t=0}^{\infty} \frac{(-1)^{g+p+q+t}(\alpha q)^{t}}{t !}\left(\begin{array}{c}
h \\
g
\end{array}\right)\left(\begin{array}{c}
b g \\
p
\end{array}\right)\left(\begin{array}{c}
a p \\
q
\end{array}\right)\left[\frac{1-e^{-\lambda x}}{1-\left(1-e^{-\lambda x}\right)}\right]^{\beta t},
$$

by using the binomial expansion in the previous equation, we obtain

$[F(x)]^{h}=\sum_{g=0}^{h} \sum_{p, q, t, \kappa, \ell_{2}=0}^{\infty} \frac{(-1)^{g+p+q+t+\ell_{2}}(\alpha q)^{t}}{t !}\left(\begin{array}{c}h \\ g\end{array}\right)\left(\begin{array}{c}b g \\ p\end{array}\right)\left(\begin{array}{c}a p \\ q\end{array}\right)\left(\begin{array}{c}\beta t+k-1 \\ k\end{array}\right)\left(\begin{array}{c}\beta t+k \\ \ell_{2}\end{array}\right) e^{-\lambda \ell_{2} x}$,

Then,

$$
[F(x)]^{h}=\sum_{g=0}^{h} \sum_{p, q, t, k, \ell_{2}=0}^{\infty} \eta_{g, p, q, t, k, \ell_{2}} e^{-\lambda \ell_{2} x}
$$

where,

$$
\eta_{g, p, q, t, k, \ell_{2}}=\frac{(-1)^{g+p+q+t+\ell_{2}}(\alpha q)^{t}}{t !}\left(\begin{array}{c}
h \\
g
\end{array}\right)\left(\begin{array}{c}
b g \\
p
\end{array}\right)\left(\begin{array}{c}
a p \\
q
\end{array}\right)\left(\begin{array}{c}
\beta t+k-1 \\
k
\end{array}\right)\left(\begin{array}{c}
\beta t+k \\
\ell_{2}
\end{array}\right) .
$$

\subsection{Quantile and median}

Quantile functions are used in theoretical aspects of probability theory, statistical applications, and simulations. Simulation methods utilize quantile function to produce simulated random variables for classical and new continuous distributions. The quantile function, say $Q(u)=F^{-1}(u)$ of $X$, is given by

$$
u=1-\left[1-\left(1-e^{-\alpha\left(e^{\lambda Q(u)}-1\right)^{\beta}}\right)^{a}\right]^{b} .
$$

After some simplifications, it reduces to the following form

$$
\mathrm{Q}(\mathrm{u})=\ln \left\{1+\left(\ln \left(1-\left(1-(1-\mathfrak{u})^{\frac{1}{b}}\right)^{\frac{1}{\alpha}}\right)^{\frac{-1}{\alpha}}\right\}^{\frac{1}{\beta}} .\right.
$$

Where, $u$ is considered as a uniform random variable on the unit interval $(0,1)$. 
In particular, the median can be derived from (3.6) be setting $u=0.5$. That is, the median is given by

$$
\text { Median }=\ln \left\{1+\left(\ln \left(1-\left(1-(0.5)^{\frac{1}{b}}\right)^{\frac{1}{a}}\right)^{\frac{-1}{\alpha}}\right\}^{\frac{1}{\beta}}\right.
$$

\subsection{Moments}

This subsection concerns with the rth moment and moment generating function for KwWE distribution. Moments are important in any statistical analysis, especially in applications. If $\mathrm{X}$ has the pdf (2.3), then its rth moment can be obtained through the following relation

$$
\mu_{r}^{\prime}=E\left(X^{r}\right)=\int_{-\infty}^{\infty} x^{r} f(x ; \Psi) d x
$$

Substituting (3.4) into (3.7) yields:

$$
\mu_{r}^{\prime}=E\left(X^{r}\right)=\sum_{i, j, k, m, \ell_{1}=0}^{\infty} \eta_{i, j, k, m, \ell_{1}} \int_{0}^{\infty} x^{r} e^{-\lambda\left(\ell_{1}+1\right) x} d x .
$$

Then, $\mu_{\mathrm{r}}^{\prime}$ becomes

$$
\mu_{r}^{\prime}=\sum_{i, j, k, m, \ell_{1}=0}^{\infty} \frac{\eta_{i, j, k, m, \ell_{1}} \Gamma(r+1)}{\left[\lambda\left(\ell_{1}+1\right)\right]^{r+1}} .
$$

Generally, the moment generating function of $\mathrm{KwWE}$ distribution is obtained through the following relation

$$
M_{X}(t)=\sum_{r=0}^{\infty} \frac{t^{r}}{r !} E\left(X^{r}\right)=\sum_{r, i, j, k, m, \ell_{1}=0}^{\infty} \frac{t^{r}}{r !} \frac{\eta_{i, j, k, m, \ell_{1}} \Gamma(r+1)}{\left[\lambda\left(\ell_{1}+1\right)\right]^{r+1}} .
$$

\subsection{Incomplete and conditional moments}

The main application of the first incomplete moment refers to the Bonferroni and Lorenz curves. These curves are very useful in economics, reliability, demography, insurance, and medicine. The answers to many important questions in economics require more than just knowing the mean of the distribution, but its shape as well. This is obvious not only in the study of econometrics but in other areas as well. The incomplete moment, say $\varphi_{s}(t)$, is given by

$$
\varphi_{s}(t)=\int_{0}^{t} x^{s} f(x ; \Psi) d x
$$

Using (2.3), then $\varphi_{s}(t)$ can be written as follows

$$
\varphi_{s}(t)=\sum_{i, j, k, m, \ell_{1}=0}^{\infty} \eta_{i, j, k, m, \ell_{1}} \int_{0}^{t} x^{s} e^{-\lambda\left(\ell_{1}+1\right) x} d x .
$$

Then, using the lower incomplete gamma function, we obtain

$$
\varphi_{s}(t)=\sum_{i, j, k, m, \ell_{1}=0}^{\infty} \eta_{i, j, k, m, \ell_{1}} \frac{v\left(s+1, \lambda\left(\ell_{1}+1\right) t\right)}{\left(\lambda\left(\ell_{1}+1\right)\right)^{s+1}},
$$

where $v(s, t)=\int_{0}^{t} x^{s-1} e^{-x} d x$ is the lower incomplete gamma function. 
Further, the conditional moment, say $\tau_{s}(t)$, is given by

$$
\tau_{s}(t)=\int_{t}^{\infty} x^{s} f(x ; \Psi) d x
$$

Hence, by using pdf (2.3), we can write

$$
\tau_{s}(t)=\sum_{i, j, k, m, \ell_{1}=0}^{\infty} \eta_{i, j, k, m, \ell_{1}} \int_{t}^{\infty} x^{s} e^{-\lambda\left(\ell_{1}+1\right) x} d x .
$$

Then using the upper incomplete gamma function, we obtain

$$
\tau_{s}(t)=\sum_{i, j, k, m, \ell_{1}=0}^{\infty} \eta_{i, j, k, m, \ell_{1}} \frac{\Gamma\left(s+1, \lambda\left(\ell_{1}+1\right) t\right)}{\left(\lambda\left(\ell_{1}+1\right)\right)^{s+1}},
$$

where $\Gamma(s, t)=\int_{t}^{\infty} x^{s-1} e^{-x} d x$ is the upper incomplete gamma function.

\subsection{Residual life function}

Several functions are defined related to the residual life. The failure rate function, mean residual life function, and the left censored mean function, also called vitality function. It is well known that these three functions uniquely determine $F(x)$, see $[13,21,28]$. Moreover, the $n$th moment of the residual life, say $m_{n}(t)=E\left[(X-t)^{n} \mid X>t\right], n=1,2, \ldots$, uniquely determine $F(x)$ (see [22]). The $n t h$ moment of the residual life of $X$ is given by

$$
m_{n}(t)=\frac{1}{R(t)} \int_{t}^{\infty}(x-t)^{n} f(x ; \Psi) d x .
$$

Applying the binomial expansion of $(x-t)^{n}$ into the above formula, we get

$$
m_{n}(t)=\frac{1}{R(t)} \sum_{i, j, k, m, \ell_{1}=0}^{\infty} \sum_{d=0}^{n}(-t)^{d}\left(\begin{array}{l}
n \\
d
\end{array}\right) \eta_{i, j, k, m, \ell_{1}} \frac{\Gamma\left(n-d+1, \lambda\left(\ell_{1}+1\right) t\right)}{\left(\lambda\left(\ell_{1}+1\right)\right)^{n-d+1}},
$$

where $\Gamma(s, t)$ is the upper incomplete gamma function.

Another interesting function is the mean residual life (MRL) function or the life expectation at age $x$ defined by $m_{1}(t)=E[(X-t) \mid X>t]$, which represents the expected additional life length for a unit which is alive at age $x$. The MRL of the KwWE distribution can be obtained by setting $n=1$ in (3.8).

Furthermore, the $n t h$ moment of the reversed residual life, say $M_{n}(t)=E\left[(X-t)^{n} \mid X \leqslant t\right]$, for $t>$ $0, n=1,2, \ldots$, uniquely determines $F(x)$ (see [22]). Hence, the $n$th moment of the reversed residual life of $\mathrm{X}$ is given by

$$
M_{n}(t)=\frac{1}{F(t)} \int_{0}^{t}(t-x)^{n} f(x) d x .
$$

Applying the binomial expansion of $(x-t)^{n}$ into the above formula, we get

$$
M_{n}(t)=\frac{1}{F(t)} \sum_{i, j, k, m, \ell_{1}=0}^{\infty} \sum_{d=0}^{n}(-1)^{n+d}(t)^{d}\left(\begin{array}{c}
n \\
d
\end{array}\right) \eta_{i, j, k, m, \ell_{1}} \frac{v\left(n-d+1, \lambda\left(\ell_{1}+1\right) t\right)}{\left(\lambda\left(\ell_{1}+1\right)\right)^{n-d+1}},
$$

where $v(s, t)$ is the lower incomplete gamma function.

The mean inactivity time or mean waiting time, also called the mean reversed residual life function, is defined by $M_{1}(t)=E[(X-t) \mid X \leqslant t]$, and it represents the waiting time elapsed since the failure of an item on condition that this failure had occurred in $(0, x)$.

\subsection{Inequality measures}

Lorenz and Bonferroni curves are the most widely used inequality measures in income and wealth distribution (see [20]). Zenga curve was presented in [26]. In this section, we will derive Lorenz, Bon- 
ferroni, and Zenga curves for the KwWE distribution. The Lorenz, Bonferroni, and Zenga curves are obtained, respectively, as

$$
\begin{aligned}
& L_{F}(x)=\frac{\int_{0}^{t} x f(x) d x}{E(X)}=\frac{\sum_{i, j, k, m, \ell_{1}=0}^{\infty} \eta_{i, j, k, m, \ell_{1}} \frac{v\left(2, \lambda\left(\ell_{1}+1\right) t\right.}{\left(\lambda\left(\ell_{1}+1\right)\right)^{2}}}{\sum_{i, j, k, m, \ell_{1}=0}^{\infty} \frac{\eta_{i, j, k, m, \ell_{1}}}{\left[\lambda\left(\ell_{1}+1\right)\right]^{2}}}, \\
& B_{F}(x)=\frac{\int_{0}^{t} x f(x) d x}{E(X) F(x)}=\frac{L_{F}(x)}{F(x)}=\frac{\sum_{i, j, k, m, \ell_{1}=0}^{\infty} \eta_{i, j, k, m, \ell_{1}} \frac{v\left(2, \lambda\left(\ell_{1}+1\right) t\right)}{\left(\lambda\left(\ell_{1}+1\right)\right)^{2}}}{\sum_{i, j, k, m, \ell_{1}=0}^{\infty} \frac{\eta_{i, j, k, m, \ell_{1}}}{\left[\lambda\left(\ell_{1}+1\right)\right]^{2}}\left[1-\left[1-\left(1-e^{\left.-\alpha\left(e^{\lambda x}-1\right)^{\beta}\right) a}\right]^{b}\right]^{2}\right.},
\end{aligned}
$$

and

$$
A_{F}(x)=1-\frac{\mu^{-}(x)}{\mu^{+}(x)^{\prime}}
$$

where

$$
\mu^{-}(x)=\frac{\int_{0}^{t} x f(x) d x}{E(X)}=\frac{\sum_{i, j, k, \ell_{1}=0}^{\infty} \eta_{i, j, k, m, \ell_{1}} \frac{v\left(2, \lambda\left(\ell_{1}+1\right) t\right)}{\left(\lambda\left(\ell_{1}+1\right)\right)^{2}}}{\sum_{i, j, k, m, \ell_{1}=0}^{\infty} \frac{\eta_{i, j, k, m, \ell_{1}}}{\left[\lambda\left(\ell_{1}+1\right)\right]^{2}}}
$$

and

$$
\mu^{+}(x)=\frac{\int_{t}^{\infty} x f(x) d x}{1-F(x)}=\frac{\sum_{i, j, k, m, \ell_{1}=0}^{\infty} \eta_{i, j, k, m, \ell_{1}} \frac{v\left(2, \lambda\left(\ell_{1}+1\right) t\right)}{\left(\lambda\left(\ell_{1}+1\right)\right)^{2}}}{\sum_{i, j, k, m, \ell_{1}=0}^{\infty} \frac{\eta_{i, j, k, m, \ell_{1}}}{\left[\lambda\left(\ell_{1}+1\right)\right]^{2}}\left[1-\left(1-e^{-\alpha\left(e^{\lambda x}-1\right)^{\beta}}\right)^{a}\right]^{b}} .
$$

\subsection{Rényi and q-entropies}

The entropy of a random variable $X$ is a measure of variation of uncertainty and has been used in many fields such as physics, engineering, and economics. The Renyi entropy in [24] is defined by

$$
I_{\delta}(X)=\frac{1}{1-\delta} \log \int_{-\infty}^{\infty} f(x ; \Psi)^{\delta} d x, \quad \delta>0 \text { and } \delta \neq 1 .
$$

By applying the binomial theory (3.1) in the pdf (2.3), then the pdf $f(x ; \Psi)^{\delta}$ can be expressed as follows

$$
f(x)^{\delta}=\sum_{i, j, k, m, \ell_{1}=0}^{\infty} W_{i, j, k, m, \ell_{1}} e^{-\lambda\left[\ell_{1}+\delta\right] x},
$$

where

$$
\begin{aligned}
W_{i, j, k, m, \ell_{1}}= & \frac{(a b \beta \lambda)^{\delta} \alpha^{k+\delta}(-1)^{i+j+k+\ell_{1}}(j+\delta)^{k}}{k !}\left(\begin{array}{c}
\delta(b-1) \\
i
\end{array}\right)\left(\begin{array}{c}
a(i+\delta)-\delta \\
j
\end{array}\right) \\
& \times\left(\begin{array}{c}
\beta(k+\delta)+\delta+m-1 \\
m
\end{array}\right)\left(\begin{array}{c}
m+\beta(k+\delta)-\delta \\
\ell_{1}
\end{array}\right) .
\end{aligned}
$$

Therefore, the Rényi entropy of $\mathrm{K} w W E$ distribution is given by

$$
I_{\delta}(X)=\frac{1}{1-\delta} \log \left[\sum_{i, j, k, m, \ell_{1}=0}^{\infty} W_{i, j, k, m, \ell_{1}} \int_{0}^{\infty} e^{\left.-\lambda\left[\ell_{1}+\delta\right)\right] x} d x\right],
$$

then,

$$
I_{\delta}(X)=\frac{1}{1-\delta} \log \left[\sum_{i, j, k, m, \ell_{1}=0}^{\infty} \frac{W_{i, j, k, m, \ell_{1}}}{\left[\lambda\left[\ell_{1}+\delta\right]\right]}\right]
$$

The q-entropy is defined by

$$
H_{q}(X)=\frac{1}{1-q} \log \left(1-\int_{-\infty}^{\infty} f(x ; \Psi)^{q} d x\right), q>0 \text { and } q \neq 1
$$


Therefore, the q-entropy of $\mathrm{K} w \mathrm{WE}$ distribution is given by

$$
H_{q}(X)=\frac{1}{1-q} \log \left\{1-\left[\sum_{i, j, k, m, \ell_{1}=0}^{\infty} \frac{W_{i, j, k, m, \ell_{1}}}{\left[\lambda\left[\ell_{1}+q\right]\right]}\right]\right\} .
$$

\subsection{The probability weighted moments}

The probability weighted moments can be obtained from the following relation

$$
\tau_{r, s}=E\left[X^{r} F(x)^{s}\right]=\int_{-\infty}^{\infty} x^{r} f(x)(F(x))^{s} d x
$$

Substituting (3.4) and (3.5) into (3.9), and replacing $h$ with $s$, leads to:

$$
\tau_{r, s}=\sum_{i, j, k, m, \ell_{1}=0}^{\infty} \sum_{g=0}^{s} \sum_{p, q, t, k, \ell_{2}=0}^{\infty} \eta_{g, p, q, t, k, \ell_{2}} \eta_{i, j, k, m, \ell_{1}} \int_{0}^{\infty} x^{r} e^{-\lambda\left[\ell_{1}+\ell_{2}+1\right] x} d x .
$$

Hence, the PWM of Kumaraswamy Weibull exponential distribution takes the following form

$$
\tau_{r, s}=\sum_{i, j, k, m, \ell_{1}=0}^{\infty} \sum_{g=0}^{s} \sum_{p, q, t, k, \ell_{2}=0}^{\infty} \frac{\eta_{g, p, q, t, k, \ell_{2}} \eta_{i, j, k, m, \ell_{1}} \Gamma(r+1)}{\left[\lambda\left[\ell_{1}+\ell_{2}+1\right]\right]^{r+1}} .
$$

\subsection{Order statistics}

Let $X_{1: n}<X_{2: n}<\cdots<X_{n: n}$ be the order statistics of a random sample of size $n$ following the Kumaraswamy Weibull exponential distribution, with parameters $a, b, \alpha, \beta$, and $\lambda$, then the pdf of the kthorder statistic (see, [8]), can be written as follows

$$
f_{k: n}(x)=-\frac{f(x)}{B(k, n-k+1)} \sum_{v=0}^{n-k}(-1)^{v}\left(\begin{array}{c}
n-k \\
v
\end{array}\right) F(x)^{v+k-1},
$$

where $B(.,$.$) is the beta function. Substituting (3.4) and (3.5) in (3.10), and replacing h$ with $v+k-1$, leads to

$$
f_{k: n}(x)=-\frac{1}{B(k, n-k+1)} \sum_{v=0}^{n-k} \sum_{i, j, k, m, \ell_{1}=0}^{\infty} \sum_{g=0}^{v+k-1} \sum_{p, q, t, k, \ell_{2}=0}^{\infty} \eta^{*} e^{-\lambda\left[\ell_{1}+\ell_{2}+1\right] x},
$$

where $\eta^{*}=(-1)^{v}\left(\begin{array}{c}n-k \\ v\end{array}\right) \eta_{i, j, k, m, \ell_{1}} \eta_{g, p, q, t, k, \ell_{2}}$.

\section{Maximum likelihood estimation}

The maximum likelihood estimates of the unknown parameters for the Kumaraswamy Weibull exponential distribution are determined based on complete samples. Let $X_{1}, \ldots, X_{n}$ be observed values from the $\mathrm{K} w W E$ distribution with set of parameters $\Psi=(a, b, \alpha, \beta, \lambda)^{\top}$. The total log-likelihood function for the vector of parameters $\Psi$ can be expressed as

$$
\begin{aligned}
\ln L(\Psi)= & n \ln a+n \ln b+n \ln \alpha+n \ln \beta+n \ln \lambda+(\beta-1) \sum_{i=1}^{n} \ln \left(e^{\lambda x_{i}}-1\right)+\lambda \sum_{i=1}^{n} x_{i} \\
& -\alpha \sum_{i=1}^{n}\left[\left(e^{\lambda x_{i}}-1\right)\right]^{\beta}+(a-1) \sum_{i=1}^{n} \ln \left[1-e^{-\alpha\left(e^{\lambda x_{i}}-1\right)^{\beta}}\right]+(b-1) \sum_{i=1}^{n} \ln \left[1-\left(1-e^{-\alpha\left(e^{\lambda x_{i}}-1\right)^{\beta}}\right)^{\alpha}\right] .
\end{aligned}
$$

The elements of the score function $\mathrm{U}(\Psi)=\left(\mathrm{U}_{\mathrm{a}}, \mathrm{U}_{\mathrm{b}}, \mathrm{U}_{\alpha}, \mathrm{U}_{\beta}, \mathrm{U}_{\lambda}\right)$ are given by

$$
\mathrm{U}_{\mathrm{a}}=\frac{\mathrm{n}}{\mathrm{a}}+\sum_{i=1}^{n} \ln \left[1-\mathrm{e}^{-\alpha\left(\mathrm{e}^{\lambda x_{i}}-1\right)^{\beta}}\right]-(\mathrm{b}-1) \sum_{i=1}^{n} \frac{\left(1-\mathrm{e}^{-\alpha\left(\mathrm{e}^{\lambda x_{i}}-1\right)^{\beta}}\right)^{\mathrm{a}} \ln \left(1-e^{-\alpha\left(\mathrm{e}^{\lambda x_{i}}-1\right)^{\beta}}\right)}{1-\left(1-e^{-\alpha\left(e^{\lambda x_{i}}-1\right)^{\beta}}\right)^{\alpha}},
$$




$$
\begin{aligned}
\mathrm{u}_{\mathrm{b}}= & \frac{\mathrm{n}}{\mathrm{b}}+\sum_{i=1}^{n} \ln \left[1-\left(1-e^{\left.\left.-\alpha\left(e^{\lambda x_{i}}-1\right)^{\beta}\right)^{\alpha}\right]}\right.\right. \\
\mathrm{u}_{\alpha}= & \frac{n}{\alpha}-\sum_{i=1}^{n}\left(e^{\lambda x_{i}}-1\right)^{\beta}+(a-1) \sum_{i=1}^{n} \frac{\left(e^{\lambda x_{i}}-1\right)^{\beta} e^{-\alpha\left(e^{\lambda x_{i}}-1\right)^{\beta}}}{1-e^{-\alpha\left(e^{\lambda x_{i}}-1\right)^{\beta}}} \\
& -a(b-1) \sum_{i=1}^{n} \frac{\left(e^{\lambda x_{i}}-1\right)^{\beta} e^{-\alpha\left(e^{\lambda x_{i}}-1\right)^{\beta}}\left(1-e^{-\alpha\left(e^{\lambda x_{i}}-1\right)^{\beta}}\right)^{a-1}}{1-\left(1-e^{-\alpha\left(e^{\lambda x_{i}}-1\right)^{\beta}}\right)^{a}}, \\
\mathrm{u}_{\beta}= & \frac{n}{\beta}+\sum_{i=1}^{n} \ln \left(e^{\lambda x_{i}}-1\right)^{\beta}-\alpha \sum_{i=1}^{n}\left(e^{\lambda x_{i}}-1\right)^{\beta} \ln \left(e^{\lambda x_{i}}-1\right) \\
& +\alpha(a-1) \sum_{i=1}^{n} \frac{\left(e^{\lambda x_{i}}-1\right)^{\beta} e^{-\alpha\left(e^{\lambda x_{i}}-1\right)^{\beta}} \ln \left(e^{\lambda x_{i}}-1\right)}{1-e^{-\alpha\left(e^{\lambda x_{i}}-1\right)^{\beta}}} \\
& -a \alpha(b-1) \sum_{i=1}^{n} \frac{\left(e^{\lambda x_{i}}-1\right)^{\beta} \ln \left(e^{\lambda x_{i}}-1\right) e^{-\alpha\left(e^{\lambda x_{i}}-1\right)^{\beta}}\left(1-e^{-\alpha\left(e^{\lambda x_{i}}-1\right)^{\beta}}\right)^{a-1}}{1-\left(1-e^{-\alpha\left(e^{\lambda x_{i}}-1\right)^{\beta}}\right)^{\alpha}}
\end{aligned}
$$

and

$$
\begin{aligned}
\mathrm{u}_{\lambda}=\frac{\mathrm{n}}{\lambda} & +(\beta-1) \sum_{i=1}^{n} \frac{x_{i} e^{\lambda x_{i}}}{e^{\lambda x_{i}}-1}-\alpha \beta \sum_{i=1}^{n} x_{i}\left(e^{\lambda x_{i}}-1\right)^{\beta-1} e^{\lambda x_{i}} \\
& +\alpha \beta(a-1) \sum_{i=1}^{n} \frac{x_{i} e^{\lambda x_{i}}\left(e^{\lambda x_{i}}-1\right)^{\beta-1} e^{-\alpha\left(e^{\lambda x_{i}}-1\right)^{\beta}}}{1-e^{-\alpha\left(e^{\lambda x_{i}}-1\right)^{\beta}}} \\
& +\sum_{i=1}^{n} x_{i}-a \alpha \beta(b-1) \sum_{i=1}^{n} \frac{x_{i} e^{\lambda x_{i}}\left(e^{\lambda x_{i}}-1\right)^{\beta-1} e^{-\alpha\left(e^{\lambda x_{i}}-1\right)^{\beta}}\left(1-e^{-\alpha\left(e^{\lambda x_{i}}-1\right)^{\beta}}\right)^{a-1}}{1-\left(1-e^{-\alpha\left(e^{\lambda x_{i}}-1\right)^{\beta}}\right)^{a}} .
\end{aligned}
$$

Then the maximum likelihood estimators of the parameters $a, b, \alpha, \beta$, and $\lambda$ are obtained by setting equations (4.1)-(4.5) to be zero and solving them. Clearly, it is difficult to solve them, therefore applying the Newton-Raphson's iteration method and using the computer package such as Maple or R or other software.

\section{Simulation study}

It is very difficult to compare the theoretical performances of the different estimates (MLE) for the KwWE distribution. Therefore, simulation is needed to compare the performances of the different methods of estimation mainly with respect to their biases, mean square errors, and Variances for different sample sizes. A numerical study is performed using Mathematica 7 software. Different sample sizes are considered through the experiments at size $\mathrm{n}=20,30,50$, and 100 .

The experiment will be repeated 1000 times. In each experiment, the estimates of the parameters will be obtained by maximum likelihood methods of estimation. The means, MSEs, and biases for the different estimators will be reported from these experiments. 
Table 2: The MLEs, baises, and MSEs of KwWE distribution.

\begin{tabular}{|c|c|c|c|c|c|c|c|c|c|}
\hline$n$ & Par & Init & MLE & Bais & MSE & Init & MLE & Bais & MSE \\
\hline & $\mathrm{a}$ & 2 & 2.0496 & 0.0496 & 0.1130 & 1.5 & 1.5531 & 0.0531 & 0.0646 \\
\hline & $\mathrm{b}$ & 0.5 & 0.5335 & 0.0335 & 0.0186 & 0.5 & 0.5239 & 0.0239 & 0.0169 \\
\hline \multirow[t]{5}{*}{20} & $\alpha$ & 0.5 & 0.5628 & 0.0628 & 0.0946 & 0.5 & 0.5334 & 0.0334 & 0.0263 \\
\hline & $\beta$ & 0.5 & 0.3789 & -0.1211 & 0.0959 & 0.5 & 0.3780 & -0.1220 & 0.0532 \\
\hline & $\lambda$ & 0.5 & 0.8185 & 0.3185 & 8.7494 & 0.5 & 0.3440 & -0.1560 & 203.1860 \\
\hline & $\mathrm{a}$ & 2 & 2.0278 & 0.0278 & 0.0746 & 1.5 & 1.5285 & 0.0285 & 0.0435 \\
\hline & $\mathrm{b}$ & 0.5 & 0.5178 & 0.0178 & 0.0099 & 0.5 & 0.5144 & 0.0144 & 0.0095 \\
\hline \multirow[t]{5}{*}{30} & $\alpha$ & 0.5 & 0.5299 & 0.0299 & 0.0192 & 0.5 & 0.5198 & 0.0198 & 0.0135 \\
\hline & $\beta$ & 0.5 & 0.3382 & -0.1618 & 0.0418 & 0.5 & 0.3516 & -0.1484 & 0.0368 \\
\hline & $\lambda$ & 0.5 & 0.7642 & 0.2642 & 3.4597 & 0.5 & 0.6287 & 0.1287 & 0.2094 \\
\hline & $\mathrm{a}$ & 2 & 2.0251 & 0.0251 & 0.0432 & 1.5 & 1.5090 & 0.0090 & 0.0242 \\
\hline & $\mathrm{b}$ & 0.5 & 0.5089 & 0.0089 & 0.0051 & 0.5 & 0.5134 & 0.0134 & 0.0056 \\
\hline \multirow[t]{5}{*}{50} & $\alpha$ & 0.5 & 0.5138 & 0.0138 & 0.0088 & 0.5 & 0.5171 & 0.0171 & 0.0078 \\
\hline & $\beta$ & 0.5 & 0.3221 & -0.1780 & 0.0390 & 0.5 & 0.3443 & -0.1557 & 0.0323 \\
\hline & $\lambda$ & 0.5 & 0.5917 & 0.0917 & 0.1203 & 0.5 & 0.5783 & 0.0783 & 0.0622 \\
\hline & $\mathrm{a}$ & 2 & 2.0054 & 0.0054 & 0.0214 & 1.5 & 1.5091 & 0.0091 & 0.0123 \\
\hline & $\mathrm{b}$ & 0.5 & 0.5075 & 0.0075 & 0.0027 & 0.5 & 0.5064 & 0.0064 & 0.0027 \\
\hline \multirow[t]{5}{*}{100} & $\alpha$ & 0.5 & 0.5111 & 0.0111 & 0.0044 & 0.5 & 0.5078 & 0.0078 & 0.0036 \\
\hline & $\bar{\beta}$ & 0.5 & 0.3148 & -0.1852 & 0.0377 & 0.5 & 0.3322 & -0.1678 & 0.0317 \\
\hline & $\lambda$ & 0.5 & 0.5458 & 0.0458 & 0.0297 & 0.5 & 0.5362 & 0.0362 & 0.0214 \\
\hline & $a$ & 2 & 2.1856 & 0.1856 & 0.4710 & 2 & 2.2533 & 0.2533 & 0.9063 \\
\hline & $\mathrm{b}$ & 1.5 & 1.5742 & 0.0742 & 0.1409 & 2 & 2.0872 & 0.0872 & 0.2610 \\
\hline \multirow[t]{5}{*}{20} & $\alpha$ & 0.5 & 0.5613 & 0.0613 & 0.0672 & 0.5 & 0.5736 & 0.0736 & 0.1787 \\
\hline & $\beta$ & 0.5 & 0.4598 & -0.0402 & 0.0422 & 0.5 & 0.4827 & -0.0173 & 0.0415 \\
\hline & $\lambda$ & 0.5 & 0.6340 & 0.1340 & 0.2020 & 0.5 & 0.5949 & 0.0949 & 0.1111 \\
\hline & $a$ & 2 & 2.1162 & 0.1162 & 0.3080 & 2 & 2.1420 & 0.1419 & 0.3793 \\
\hline & $\mathrm{b}$ & 1.5 & 1.5452 & 0.0452 & 0.0815 & 2 & 2.0750 & 0.0750 & 0.1608 \\
\hline \multirow[t]{5}{*}{30} & $\alpha$ & 0.5 & 0.5350 & 0.0350 & 0.0259 & 0.5 & 0.5448 & 0.0448 & 0.0356 \\
\hline & $\bar{\beta}$ & 0.5 & 0.4295 & -0.0705 & 0.0232 & 0.5 & 0.4693 & -0.0307 & 0.0230 \\
\hline & $\lambda$ & 0.5 & 0.5689 & 0.0688 & 0.0570 & 0.5 & 0.5678 & 0.0678 & 0.0516 \\
\hline & $a$ & 2 & 2.0708 & 0.0708 & 0.1373 & 2 & 2.0924 & 0.0924 & 0.1980 \\
\hline & $\mathrm{b}$ & 1.5 & 1.5312 & 0.0312 & 0.0477 & 2 & 2.0320 & 0.0320 & 0.0860 \\
\hline \multirow[t]{5}{*}{50} & $\alpha$ & 0.5 & 0.5209 & 0.0209 & 0.0127 & 0.5 & 0.5199 & 0.0199 & 0.0152 \\
\hline & $\beta$ & 0.5 & 0.4197 & -0.0803 & 0.0170 & 0.5 & 0.4474 & -0.0526 & 0.0146 \\
\hline & $\lambda$ & 0.5 & 0.5448 & 0.0448 & 0.0283 & 0.5 & 0.5323 & 0.0323 & 0.0237 \\
\hline & $a$ & 2 & 2.0353 & 0.0353 & 0.0626 & 2 & 2.0305 & 0.0305 & 0.0809 \\
\hline & $\mathrm{b}$ & 1.5 & 1.5135 & 0.0135 & 0.0240 & 2 & 2.0236 & 0.0236 & 0.0381 \\
\hline \multirow[t]{3}{*}{100} & $\alpha$ & 0.5 & 0.5094 & 0.0094 & 0.0059 & 0.5 & 0.5118 & 0.0118 & 0.0055 \\
\hline & $\beta$ & 0.5 & 0.4056 & -0.0944 & 0.0140 & 0.5 & 0.4378 & -0.0622 & 0.0088 \\
\hline & $\lambda$ & 0.5 & 0.5203 & 0.0203 & 0.0127 & 0.5 & 0.5188 & 0.0188 & 0.0091 \\
\hline
\end{tabular}

\section{Data analysis}

In this section, one real data set are analyzed to illustrate the merit of $\mathrm{K} w \mathrm{WE}$ distribution compared to some sub-models; namely, Weibull exponential (WE) [23], beta Weibull (BW) [7], and Weibull Weibull (WW) [1] distributions. 
We obtain the MLE and their corresponding standard errors (in parentheses) of the model parameters. To compare the distribution models, we consider criteria like; minus of log-likelihood function $(-2 \ln \mathrm{L})$, Kolmogorov-Smirnov (K-S) statistic, Akaike information criterion (AIC), the correct Akaike information criterion (CAIC), Bayesian information criterion (BIC), Hannan Quinn information criterion (HQIC) and p-value. However, the better distribution corresponds to the smaller values of $-2 \ln \mathrm{L}, \mathrm{AIC}, \mathrm{BIC}, \mathrm{CAIC}$, HQIC, K-S criteria and biggest p-value. Furthermore, we plot the histogram for each data set and the estimated pdf of the KwWE, WE, BW, and WW models. Moreover, the plots of empirical cdf of the data sets and estimated pdf of KwWE, WE, BW, and WW models are displayed in Figures 3 and 4, respectively.

The data set have been obtained from [18] and represents thirty successive values of March precipitation (in inches) in Minneapolis/St Paul. The data are as follows:

$0.77,1.74,0.81,1.20,1.95,1.20,0.47,1.43,3.37,2.20,3.00,3.09,1.51,2.10,0.52,1.62,1.31,0.32,0.59,0.81$, $2.81,1.87,1.18,1.35,4.75,2.48,0.96,1.89,0.90,2.05$.

Table 3 gives MLEs of parameters of the KwWE and their standard error (S.E). The values of the log-likelihood functions, AIC, CAIC, BIC, HQIC, K-S and p-value are presented in Table 4.

Table 3: The MLEs and S.E of the model parameters for the data set.

\begin{tabular}{|c|c|c|c|c|c|}
\hline Model & \multicolumn{5}{|l|}{ MLEs and S. E } \\
\hline $\operatorname{KWWE}(a, b, \alpha, \beta, \lambda)$ & $8.784(0.85748)$ & $5.397(0.772)$ & $5.964(0.194)$ & $0.469(0.015)$ & $0.037(0.287)$ \\
\hline $\mathrm{WE}(\alpha, \beta, \lambda)$ & - & - & $35.218(0.26269)$ & $1.69(0.234)$ & $0.06(0.044)$ \\
\hline $\operatorname{BW}(a, b, \alpha, \beta)$ & $25.851(1.533)$ & $15.276(0.787)$ & $0.884(0.201)$ & $0.335(0.027)$ & - \\
\hline $\mathrm{WW}(\alpha, \beta, \lambda, \gamma)$ & $39.853(0.414)$ & $3.154(0.518)$ & $0.196(0.102)$ & $0.5(0.072)$ & - \\
\hline
\end{tabular}

Table 4: The values of -2LnL , AIC, BIC, CAIC, HQIC, K-S, and p-value for the data set.

\begin{tabular}{|c|c|c|c|c|c|c|c|}
\hline Distribution & -2LnL & AIC & CAIC & BIC & HQIC & K-S & p-value \\
\hline KwWE & 107.911 & 117.911 & 120.411 & 115.296 & 120.152 & 0.06103 & 0.99988 \\
\hline WE & 112.107 & 118.107 & 119.031 & 116.539 & 119.452 & 0.0753 & 0.996 \\
\hline BW & 149.897 & 157.897 & 157.326 & 159.497 & 161.522 & 0.07958 & 0.9913 \\
\hline WW & 138.194 & 146.194 & 145.623 & 147.794 & 149.819 & 0.07549 & 0.99554 \\
\hline
\end{tabular}

We find that the KwWE distribution with five parameters provides a better fit than their special submodels. It has the smallest K-S, AIC, CAIC, BIC, and HQIC values among those considered here. Plots of the fitted densities and the histogram are given in Figures 3 and 4, respectively.

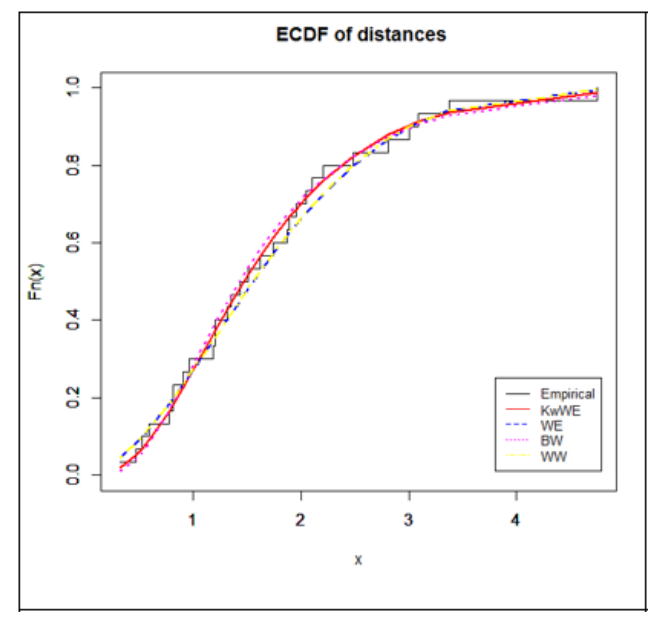

Figure 3: Estimated cumulative densities for the data set.

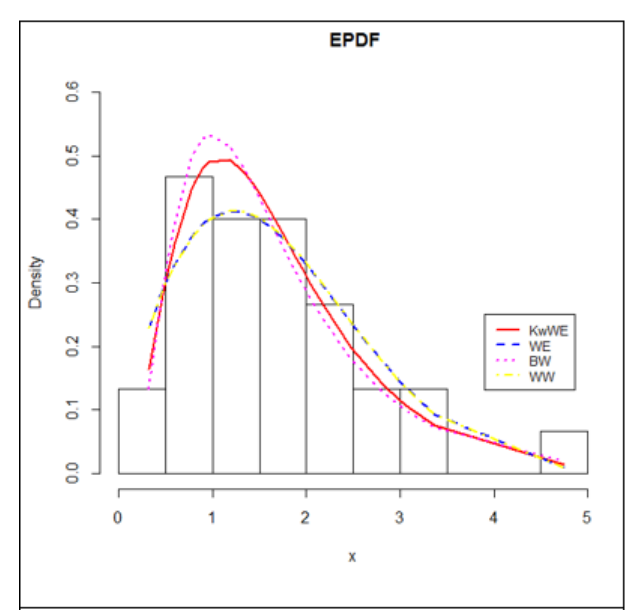

Figure 4: Estimated densities of models for the data set. 


\section{Conclusion}

We have introduced a new five-parameter Kumaraswamy Weibull exponential distribution and study its different properties in this paper. It is observed that the proposed $\mathrm{K} w \mathrm{WE}$ distribution has several desirable properties. The KwWE distribution covers some existing distributions and contains some new distributions. The practical importance of the new distribution was demonstrated in two applications where the $\mathrm{K} w \mathrm{WE}$ distribution provided better fitting in comparison with several other former lifetime distributions. Application showed that the KwWE model can be used rather than other known distributions.

\section{Acknowledgment}

This work was supported by the Deanship of Scientific Research (DSR), King AbdulAziz University, Jeddah, under grant No. (D-073-305-1439). The authors, therefore, gratefully acknowledge the DSR technical and financial support.

\section{References}

[1] T. H. M. Abouelmagd, S. Al-mualim, M. Elgarhy, A. Z. Afify, M. Ahmad, Properties of the four-parameter weibull distribution and its applications, Pak. J. Stat., 33 (2017), 449-466. 6

[2] C. Alexander, G. M. Cordeiro, E. M. M. Ortega, J. M. Sarabia, Generalized beta generated distributions, Comput. Statist. Data Anal., 56 (2012), 1880-1897. 1

[3] M. Alizadeh, M. Emadi, M. Doostparast, G. M. Cordeiro, E. M. M. Ortega, R. R. Pescim, A new family of distributions: the Kumaraswamy odd log-logistic, properties and applications, Hacet. J. Math. Stat., 44 (2015), 1491-1512. 1

[4] A. Alzaatreh, C. Lee, F. Famoye, A new method for generating families of continuous distributions, Metron, 71 (2013), 63-79. 1

[5] M. Bourguignon, R. B. Silva, G. M. Cordeiro, The Weibull-G family of probability distributions, J. Data Sci., 12 (2014), 53-68. 1

[6] G. M. Cordeiro, M. de Castro, A new family of generalized distributions, J. Stat. Comput. Simul., 81 (2011), $883-898$. 1

[7] G. M. Cordeiro, E. M. M. Ortega, S. Nadarajah, The Kumaraswamy Weibull distribution with application to failure data, J. Franklin Inst., 347 (2010), 1399-1429. 6

[8] H. A. David, Order Statistics, Second edition, John Wiley \& Sons, Inc., New York, (1981). 3.9

[9] M. Elgarhy, A. S. Hassan, M. Rashed, Garhy-Generated Family of Distributions with Application, Math. Model. Theory, 6 (2016), 1-15. 1

[10] M. Elgarhy, M. A. U. Haq, G. Özel Kadilar, A. Nasir, A New Exponentiated Extended Family of Distributions with Applications, Gazi University Journal of Science (GUJS), 30 (2017), 101-115. 1

[11] M. Elgarhy, M. Shakil, B. M. Golam Kibria, Exponentiated Weibull-exponential distribution with applications, Appl Appl Math., 12 (2017), 710-725.

[12] N. Eugene, C. Lee, F. Famoye, Beta-normal distribution and its applications, Comm. Statist. Theory Methods, 31 (2002), 497-512. 1

[13] R. C. Gupta, On characterization of distributions by conditional expectations, Comm. Statist., 4 (1975), 99-103. 3.5

[14] A. S. Hassan, M. Elgarhy, Kumaraswamy Weibull-generated family of distributions with applications, Adv. Appl. Stat., 48 (2016), 205-239. 1, 2

[15] A. S. Hassan, M. Elgarhy, A New Family of Exponentiated Weibull-Generated Distributions, Int. J. Math. Appl., 4 (2016), 135-148. 1

[16] A. S. Hassan, M. Elgarhy, M. Shakil, Type II Half Logistic Family of Distributions with Applications, Pak. J. Stat. Oper. Res., 13 (2017), 245-264. 1

[17] A. S. Hassan, S. E. Hemeda, The additive Weibull-g family of probability distributions, Int. J. Math. Appl., 4 (2016), 151-164. 1

[18] D. Hinkley, On quick choice of power transformations, J. R. Stat. Soc. Ser. C Appl. Stat., 26 (1977), 67-69. 6

[19] M. C. Jones, Families of distributions arising from the distributions of order statistics, Test, 13 (2004), 1-43. 1

[20] C. Kleiber, On Lorenz Order with in Parametric Families of Income Distributions, Sankhyā Ser. B, 61 (1999), $514-517$. 3.6

[21] S. Kotz, D. N. Shanbhag, Some new approaches to probability distributions, Adv. in Appl. Probab., 12 (1980), $903-921$. 3.5

[22] J. Navarro, M. Franco, J. M. Ruiz, Characterization through moments of the residual life and conditional spacing, Sankhyā Ser. A, 60 (1998), 36-48. 3.5, 3.5 
[23] P. E. Oguntunde, O. S. Balogun, H. I. Okagbue, S. A. Bishop, The Weibull-Exponential Distribution: Its Properties and Applications, J. Appl. Sci., 15 (2015), 1305-1311. 6

[24] A. Rényi, On measures of entropy and information, 4th Berkeley Sympos. Math. Statist. and Prob. (Univ. California Press, Berkeley), 1 (1961), 547-561. 3.7

[25] M. M. Ristić, N. Balakrishnan, The gamma-exponentiated exponential distribution, J. Stat. Comput. Simul., 82 (2012), 1191-1206. 1

[26] M. Zenga, Inequality curve and inequality index based on the ratios between lower and upper arithmetic means, Statistica e Applicazioni, 4 (2007), 3-27. 3.6

[27] K. Zografos, N. Balakrishnan, On families of beta- and generalized gamma-generated distributions and associated inference, Stat. Methodol., 6(2009), 344-362. 1

[28] P. Zoroa, J. M. Ruiz, J. A. Marín, characterization based on conditional expectations, Comm. Statist. Theory Methods, 19 (1990), 3127-3135. 3.5 